Authors

Jianfei Liu, Xiongbin Wu, William J. Emery, Lan Zhang, Chuan Li, and Ketao Ma 


\title{
Direction of Arrival Estimation and Sensor Array Error Calibration Based on Blind Signal Separation
}

\begin{abstract}
We consider estimating the direction of arrival (DOA) in the presence of sensor array error. In the proposed method, a blind signal separation method, the Joint Approximation and Diagonalization of Eigenmatrices (JADE) algorithm, is implemented to separate the signal vector and the mixing matrix consisting of the array manifold matrix and the sensor array error matrix. Based on a new mixing matrix and the reconstruction of the array output vector of each individual signal, we propose a novel DOA estimation method and sensor array error calibration procedure. This method is independent of array phase errors and performs well against difference of SNR of signals. Numerical simulations verify the effectiveness of the proposed method.
\end{abstract}

Index Terms-Directions of arrival (DOA), sensor array error calibration, Joint Approximation and Diagonalization of Eigenmatrices (JADE).

\section{INTRODUCTIONEquation Section (Next)}

Direction of arrival (DOA) estimation has gained increasing attention due to its significance in practical applications such as sonar, radar and mobile communications [1]. In particular, various super-resolution algorithms like MUSIC [2], ESPRIT [3] and BCS [4] have demonstrated excellent DOA estimation performance. Most of these array signal processing techniques presume that the array manifold vector is precisely known. Consequently, the presence of sensor array error will seriously degrade the performance of the DOA estimation, resulting in spurious directions and poor angular resolution.

In recent decades, some algorithms have been developed for estimating DOAs of signals and array errors [5] [6] [7] [8] [9] [10]. The method in [5] estimates array gain and phase errors and the DOAs simultaneously, but it is limited to small array perturbations and suffers from suboptimal convergence. Although [6], [7], [8] do not require an iteration process, these methods are confined to linear arrays. In [9] an eigenstructure approach is used to estimate DOAs and array error for nonlinear arrays. This study is based on the eigen decomposition of the covariance matrix which is derived from the dot product of a received vector with its conjugate. But it requires at least two signals which are spatially far from each other. Cao et al. estimate the DOAs using the eigen decomposition of the Hadamard product derived from a covariance matrix with its conjugate [10]. The approach proposed here subtracts the one component, which can cause a ridge close to the diagonal line of the spatial spectrum. Hence, it doesn't require that the two signals are spatially far apart. This strategy, however, needs to decompose the covariance matrix many times to calculate the power of the signals. Moreover, both [9] 
and [10] require at least $K(K-1)+1$ receiving sensors for the DOA estimation of $K$ signals.

In this letter, we utilize a novel strategy to solve the problem of estimating signal DOAs and the associated sensor array error. A blind signal separation method, the Joint Approximation and Diagonalization of Eigenmatrices (JADE) [11] algorithm, is implemented to separate the signal vector and mixing matrix. Gain errors are estimated by a conventional method in [12]. The array output vector of each individual signal is reconstructed by the estimated mixing matrix and the signal vector. Based on a new mixing matrix derived from the estimated sensor array error matrix, a novel two-dimensional spatial spectrum is formed. By locating the peak of the spatial spectrum, the DOAs are estimated. Then the phase errors are obtained from the mixing matrix and estimated DOAs. The presented method is independent of array phase errors and performs well against difference of SNR of signals. Additionally, this method only requires $K+1$ sensors for the DOA estimation of $K$ signals.

\section{SIGNAL MODEL}

Given that $K$ narrowband far-field signals impinge on an $M$-sensor array from directions $\boldsymbol{\theta}=\left[\theta_{1}, \theta_{2}, \ldots, \theta_{K}\right]^{T}$, where $(\cdot)^{T}$ represents the transpose operation. The array output vector $\mathbf{X}(t)$ is described by

$$
\mathbf{X}(t)=\mathbf{\Gamma A S}(t)+\mathbf{N}(t), \quad t=1,2, \ldots, L
$$

and $\mathbf{S}(t)=\left[\mathbf{s}_{1}(t), \mathbf{s}_{2}(t), \ldots, \mathbf{s}_{K}(t)\right]^{T}$ denotes the vector of zero-mean signals. $\mathbf{N}(t)$ represents the vector of zero-mean and $\sigma_{n}^{2}$ variance noise, which is supposed to be a spatially white Gaussian random process. Furthermore, we suppose that the first sensor of the array is the reference sensor and its location is fixed to the origin of co-ordinates.

A denotes the ideal array manifold matrix, which is given by

$$
\begin{gathered}
\mathbf{A}=\left[\mathbf{a}\left(\theta_{1}\right), \mathbf{a}\left(\theta_{2}\right), \ldots, \mathbf{a}\left(\theta_{K}\right)\right] \\
\mathbf{a}\left(\theta_{k}\right)=\left[1, e^{-j \frac{2 \pi}{\lambda} d_{2 k}}, \ldots, e^{-j \frac{2 \pi}{\lambda} d_{M k}}\right]^{T} \\
d_{m k}=\left[\begin{array}{ll}
x_{m} & y_{m}
\end{array}\right]\left[\sin \theta_{k} \cos \theta_{k}\right]^{T}
\end{gathered}
$$

where $\lambda$ denotes the center wavelength of the signals. $\left(x_{m}, y_{m}\right)$ are co-ordinates of the $m$ th sensor and $d_{m k}$ is the spatial distance between the $m$ th sensor and the first sensor in the direction of the $k$ th signal.

$\boldsymbol{\Gamma}=\operatorname{diag}\left[1, \gamma_{2}, \ldots, \gamma_{M}\right]$ is an $M \times M$ diagonal array error matrix. In addition, the array error of the $m$ th sensor can be expressed as

$$
\gamma_{m}=g_{m} e^{j \beta_{m}}
$$

where $g_{m}$ and $\beta_{m}$ are gain error and phase error of the $m$ th sensor, respectively.

Finally, define the mixing matrix as 


$$
\mathbf{B}=\left[\mathbf{b}\left(\theta_{1}\right), \mathbf{b}\left(\theta_{2}\right), \ldots, \mathbf{b}\left(\theta_{K}\right)\right]=\mathbf{\Gamma} \mathbf{A}
$$

We suppose that the signals are non-Gaussian and uncorrelated with each other. All the signals are independent of the noise have different DOAs and have different DOAs.

\section{THE NEW METHOD}

\section{A. Mixing Matrix and Signal Vector Estimation}

The JADE algorithm has proven to be a successful algorithm to calculate the mixing matrix and the signal vector in terms of the computational complexity and estimation accuracy [13]. We apply the JADE algorithm for the signal vector $\mathbf{S}(t)$ and mixing matrix $\mathbf{B}$ estimation based on the array output vector $\mathbf{X}(t)$ and the number of signals $K$. The detail implementation of this algorithm is discussed by [11]. The JADE algorithm statistically calculates a whitening matrix $\hat{\mathbf{W}}$ and a unitary matrix $\hat{\mathbf{U}}$. Thus, the mixing matrix can be estimated as

$$
\hat{\mathbf{B}}=\hat{\mathbf{W}}^{\dagger} \hat{\mathbf{U}}
$$

where $(\cdot)^{\dagger}$ is the pseudo-inverse and the signal vector is obtained as

$$
\hat{\mathbf{S}}(t)=\hat{\mathbf{U}}^{H} \hat{\mathbf{W}} \mathbf{X}(t)
$$

where $(\cdot)^{H}$ denotes the Hermitian transpose.

\section{B. Gain Error Calibration}

The sample array covariance matrix is computed from the $L$ data samples

$$
\hat{\mathbf{R}}=\frac{1}{L} \sum_{t=1}^{L} \mathbf{X}(t) \mathbf{X}^{H}(t)=\sum_{m=1}^{M} \mu_{m} \mathbf{h}_{m} \mathbf{h}_{m}^{H}
$$

where $\left[\mu_{1}, \mu_{2}, \ldots, \mu_{M}\right]$ is the eigenvalues in descending order and $\left[\mathbf{h}_{1}, \mathbf{h}_{2} \ldots, \mathbf{h}_{M}\right]$ is the eigenvectors of $\hat{\mathbf{R}}$.

Then, the noise variance $\sigma_{n}^{2}$ can be calculated from the $M-K$ smallest eigenvalues of $\hat{\mathbf{R}}$

$$
\hat{\sigma}_{n}^{2}=\frac{1}{M-K} \sum_{m=K+1}^{M} \mu_{m}
$$

Let $\hat{R}(m, m)$ denote the $m$ th diagonal element of $\hat{\mathbf{R}}$. Gain errors can be estimated as [12]

$$
\hat{g}_{m}=\sqrt{\frac{\hat{R}(m, m)-\hat{\sigma}_{n}^{2}}{\hat{R}(1,1)-\hat{\sigma}_{n}^{2}}}
$$




\section{DOA Estimation}

Gain errors are not considered in the following discussion, because they can be estimated by (11). Consider two of the $K$ signals from the directions $\theta_{k}$ and $\theta_{l}$, where $l, k=1,2, \ldots, K$ and $l \neq k$. According to (6) and (7), the mixing matrix of the $k$.th and $l$ th signal can be written as

$$
\begin{aligned}
\hat{\mathbf{b}}\left(\theta_{l}\right) & =\boldsymbol{\Gamma} \mathbf{a}\left(\theta_{l}\right) \\
\hat{\mathbf{b}}\left(\theta_{k}\right) & =\boldsymbol{\Gamma} \mathbf{a}\left(\theta_{k}\right)
\end{aligned}
$$

Let $\hat{\boldsymbol{\Gamma}}_{l}$. and $\hat{\boldsymbol{\Gamma}}_{k}$ denote the estimation of $\boldsymbol{\Gamma}$ with (12) and (13), respectively. The sensor array error matrix can be estimated as

$$
\hat{\boldsymbol{\Gamma}}_{l}=\operatorname{diag}\left[\hat{\mathbf{b}}\left(\theta_{l}\right) \odot \mathbf{a}^{*}\left(\theta_{l}\right)\right]
$$

or

$$
\hat{\boldsymbol{\Gamma}}_{k}=\operatorname{diag}\left[\hat{\mathbf{b}}\left(\theta_{k}\right) \odot \mathbf{a}^{*}\left(\theta_{k}\right)\right]
$$

where $\odot$ and $(\cdot)^{*}$ represent the Hadamard product and the conjugate operation, respectively.

Define a new mixing matrix as $\mathbf{c}_{k}\left(\theta_{l}, \theta_{k}\right)=\hat{\boldsymbol{\Gamma}}_{l} \mathbf{a}\left(\theta_{k}\right)$, and insert (14) into it

$$
\begin{aligned}
\mathbf{c}_{k}\left(\theta_{l}, \theta_{k}\right) & =\left\{\operatorname{diag}\left[\hat{\mathbf{b}}\left(\theta_{l}\right) \odot \mathbf{a}^{*}\left(\theta_{l}\right)\right]\right\} \cdot \mathbf{a}\left(\theta_{k}\right) \\
& =\hat{\mathbf{b}}\left(\theta_{l}\right) \odot \mathbf{a}^{*}\left(\theta_{l}\right) \odot \mathbf{a}\left(\theta_{k}\right)
\end{aligned}
$$

Consider the signal model in (1) with no noise. The array output vector of the $k$ th signal is defined as

$$
\mathbf{x}_{k}(t)=\hat{\boldsymbol{\Gamma}}_{l} \mathbf{a}\left(\theta_{k}\right) \mathbf{s}_{k}(t)=\mathbf{c}_{k}\left(\theta_{l}, \theta_{k}\right) \mathbf{s}_{k}(t)
$$

Based on the estimated mixing matrix and signal vector of the $k$ th signal by (7) and (8), respectively, $\mathbf{x}_{k}(t)$ can be reconstructed as

$$
\hat{\mathbf{x}}_{k}(t)=\hat{\mathbf{b}}\left(\theta_{k}\right) \hat{\mathbf{s}}_{k}(t), \quad t=1,2, \ldots, L
$$

The covariance matrix of $\hat{\mathbf{x}}_{k}(t)$ is estimated as

$$
\begin{gathered}
\hat{\mathbf{R}}_{k}=\frac{1}{L} \sum_{t=1}^{L} \hat{\mathbf{x}}_{k}(t) \hat{\mathbf{x}}_{k}^{H}(t)=\hat{E}_{s}(k) \Lambda_{s} \hat{E}_{s}^{H}(k) \\
+\hat{E}_{n}(k) \Lambda_{n} \hat{E}_{n}^{H}(k)
\end{gathered}
$$

where $\hat{E}_{s}(k)$ and $\hat{E}_{n}(k)$ represent the signal subspace and the noise subspace of the $k$ th signal, respectively. $\Lambda_{s}$ and $\Lambda_{n}$ denote the diagonal array of the largest eigenvalues and the $M-1$ smallest eigenvalues of $\hat{\mathbf{R}}_{k}$, respectively. 
As a consequence, we can present a novel DOA estimation method based on subspace principle. Since the noise subspace is orthogonal to the new mixing matrix $\mathbf{c}_{k}\left(\theta_{l}, \theta_{k}\right)$, a new two-dimensional spatial spectrum can be described by

$$
P_{k}\left(\theta^{\prime}, \theta\right)=\left\|\hat{E}_{n}^{H}(k) \mathbf{c}_{k}\left(\theta^{\prime}, \theta\right)\right\|_{2}^{-2}
$$

where $\|\cdot\|_{2}$ represents the 2-norm of a vector.

Let $[\cdot]_{k}$ denote the DOA estimated by the reconstruction of the $k$ th signal. By locating the peak from the spatial spectrum $P_{k}\left(\theta^{\prime}, \theta\right)$, we can estimate the DOAs as

$$
\left[\hat{\theta}_{k}, \hat{\theta}_{l}\right]_{k}=\arg \max _{\theta^{\prime}, \theta} P_{k}\left(\theta^{\prime}, \theta\right)
$$

On the other hand, the $l$ th signal can also be reconstructed to calculate the spatial spectrum $P_{l}\left(\theta^{\prime}, \theta\right)$, then the DOAs are estimated as

$$
\left[\hat{\theta}_{k}, \hat{\theta}_{l}\right]_{l}=\arg \max _{\theta^{\prime}, \theta} P_{l}\left(\theta^{\prime}, \theta\right)
$$

Based on the above analysis, the DOA of the $k$ th signal can be estimated with the rest $K-1$ signals

$$
\hat{\theta}_{k}=\frac{1}{K(K-1)} \sum_{m=1}^{K} \sum_{n=1 ; m \neq n}^{K}\left\{\left[\theta_{k}\right]_{m}+\left[\theta_{k}\right]_{n}\right\}
$$

\section{Phase Error Calibration}

As the DOAs have been estimated by (23), we can estimate phase errors by (14) and (15) with each pair of $\left[\hat{\theta}_{k}, \hat{\theta}_{l}\right]$, where $l, k=1,2, \ldots, K$ and $l \neq k$. For all the $K$ signals, there are $\frac{K(K-1)}{2}$ pairs of DOAs. Hence, the phase errors $\boldsymbol{\beta}=\left[1, \beta_{2}, \ldots, \beta_{M}\right]^{T}$ are obtained as

$$
\hat{\boldsymbol{\beta}}=\angle\left\{\frac{1}{K(K-1)} \sum_{m=1}^{K} \sum_{n=1 ; m \neq n}^{K}\left[\hat{\mathbf{b}}\left(\theta_{m}\right) \odot \mathbf{a}^{*}\left(\hat{\theta}_{n}\right)\right]\right\}
$$

where $\angle\{\cdot\}$ denotes the phase of a complex number.

In addition, phase errors can also be derived by the conventional algorithm in [5], which is used by [9] and [10].

\section{E. Discussion}

First, we note that the JADE algorithm is applicable for mixing matrix estimation with non-Gaussian signals, but also note that there are many non-Gaussian signal models in radar systems. 
Four of the five famous radar detection models described in [14] are non-Gaussian signal models [8]. The method presented also has value as a practical application.

Second, for a linear array, the spatial distance is $d_{m k}=x_{m} \sin \theta_{k}$. Consider two pairs of DOAs $\left[\theta_{1}, \theta_{2}\right] \neq\left[\theta_{3}, \theta_{4}\right]$ and $\left[\theta_{1}, \theta_{2}\right] \neq\left[\theta_{4}, \theta_{3}\right], \quad \mathbf{c}_{2}\left(\theta_{1}, \theta_{2}\right)$ may be equal to $\mathbf{c}_{4}\left(\theta_{3}, \theta_{4}\right)$ because $\sin \theta_{1}-\sin \theta_{2}=\sin \theta_{3}-\sin \theta_{4}$. Hence, this method is applicable to nonlinear arrays. In addition, if four sensors of an array are located on each vertex of a square with its side no more than $\frac{\lambda}{4}$, respectively, the DOA estimation using the presented method is unambiguous [9].

Third, the proposed method only requires $K+1$ sensors to estimate $K$ signals while [9] and [10] requires at least $K(K-1)+1$ sensors. From Table I we know that as $K$ increases, $M$, as required by [9] and [10] increases more rapidly than that of the proposed method. Thus, this will increase the complexity of the receiving system, which means more sensors, more receiving channels and larger array.

TABLE 1The minimum sensors required for DOA estimation against number of signals

\begin{tabular}{|c|c|c|c|c|c|c|c|c|c|}
\hline$K$ & 2 & 3 & 4 & 5 & 6 & 7 & 8 & 9 & 10 \\
\hline$M($ Our Method) & 3 & 4 & 5 & 6 & 7 & 8 & 9 & 10 & 11 \\
\hline$M([9]$ and [10]) & 3 & 7 & 13 & 21 & 31 & 43 & 57 & 73 & 91 \\
\hline
\end{tabular}

\section{NUMERICAL SIMULATIONS}

We carry out some representative simulations to verify the validity of the approach presented. The DOAs of the signals are within the region $-90^{\circ}$ to $90^{\circ}$ and we assume that two signals do exist. The array gain-phase errors of the sensors are generated as $g_{m}=1+\sqrt{12} \sigma_{g} \alpha_{m}$ and $\beta_{m}=\sqrt{12} \sigma_{\beta} \eta_{m}$, respectively. In addition, $\alpha_{m}$ and $\eta_{m}$ are independent and random variables with uniform distributions over $[-0.5,0.5]$. The standard deviations of $g_{m}$ and $\beta_{m}$ are $\sigma_{g}$ and $\sigma_{\beta}$, respectively.

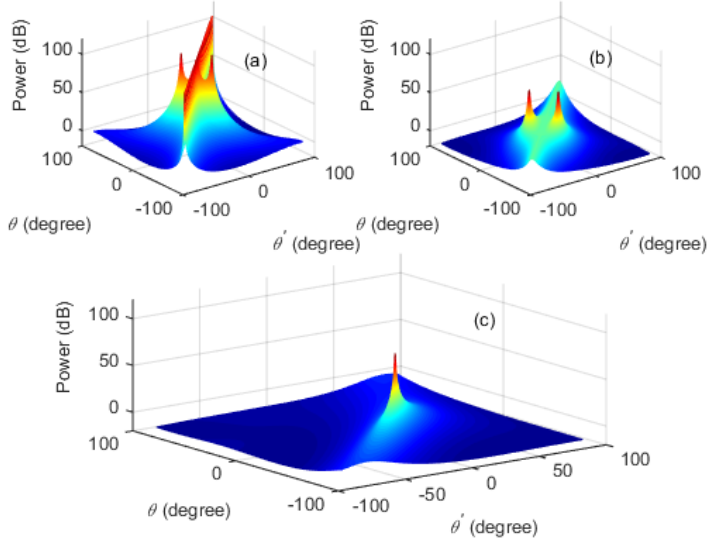

Fig. 1 Spatial spectrum of the three methods. (a) Spatial spectrum of [9].

(b) Spatial spectrum of [10]. (c) Spatial spectrum of the new Method.

In the simulations below we have, $\sigma_{g}=0.1$, the samples are $L=500$ and the number of Monte 
Carlo trials is 500 . We use the following sensor configuration to get the unambiguous DOA estimation [9]: there are seven sensors with locations at $(0,0),\left(\frac{\lambda}{4}, 0\right),\left(\frac{\lambda}{4}, \frac{\lambda}{4}\right),\left(0, \frac{\lambda}{4}\right)$, $\left(\frac{\lambda}{4} \cos 80^{\circ}, \frac{\lambda}{4} \sin 80^{\circ}\right),\left(\frac{\lambda}{4} \cos 160^{\circ}, \frac{\lambda}{4} \sin 160^{\circ}\right),\left(\frac{\lambda}{4} \cos 240^{\circ}, \frac{\lambda}{4} \sin 240^{\circ}\right)$.

The methods discussed in [9] and [10] are selected for a performance comparison. As the three methods apply the same gain error calibration method in [12], we don't discuss it in the experiments below. Both [9] and [10] implement the same phase error calibration method presented in [5], and we compare it with the new calibration method presented by (24).

Fig. 1 shows the two-dimensional spatial spectrum of the three methods, where DOAs are $\left[-25^{\circ}, 0^{\circ}\right]$, SNR of signals is $20 \mathrm{~dB}$ and $\sigma_{\beta}=40^{\circ}$. There is only one distinct peak in the spectrum of the new method compared with the spectrum in [9] and [10]. By locating the peak of the spectrum, the DOAs are estimated.

Fig. 2(a) shows RMSE (root mean square error) of the DOA estimation and array error calibration against $\sigma_{\beta}$, where DOAs are $\left[-25^{\circ}, 0^{\circ}\right]$ and SNR is $20 \mathrm{~dB}$. This demonstrates that the three methods are independent of array phase error, and reveals that the new approach performs better. It can be clearly seen that this new method is efficient and achieves higher accuracy.

Fig. 2(b) displays RMSE against DOA Separation, where DOAs are $\left[\theta_{1}, \theta_{2}\right]$, SNR is $20 \mathrm{~dB}$ and $\sigma_{\beta}=40^{\circ} . \theta_{1}=-25^{\circ}$ and DOA separation is described by $\Delta \theta=\theta_{2}-\theta_{1} . \Delta \theta$ starts from $15^{\circ}$ because [9] fails when $\Delta \theta$ is less than $15^{\circ}$. Simulation curves in Fig. 2(b) indicate that [9] performs poorly while the DOA separation is small. Both the new approach and [10] perform better as the DOA separation increases. Furthermore, the new calibration method outperforms [5].

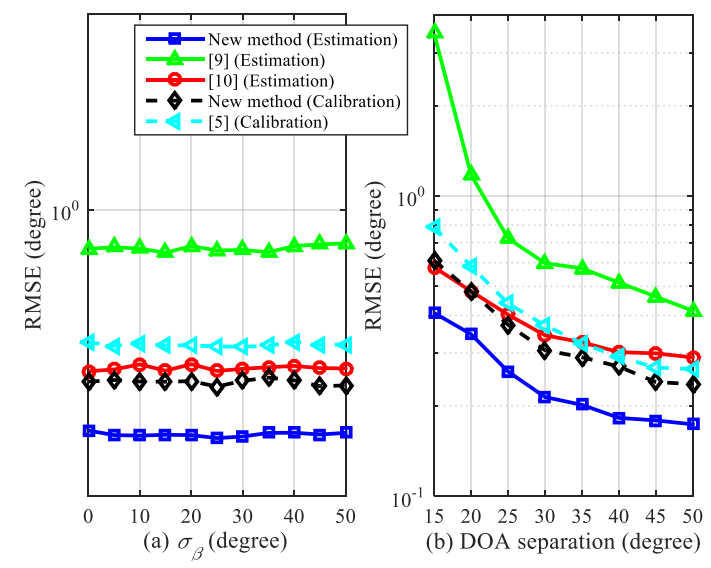

Fig. 2. RSME against $\sigma_{\beta}$ and DOA Separation. (a) RSME against $\sigma_{\beta}$. (b)

RSME against DOA Separation.

Fig. 3(a) displays RMSE against SNR, where $\sigma_{\beta}=40^{\circ}$. As seen in Fig. 3(a), the performance of the three methods improves as the SNR increases. In addition, the new estimation outperforms [9] and [10], and the new calibration method performs better than [5]. Here we also show the $95 \%$ 
confidence interval $(\mathrm{CI})$ of the bias and variance of the DOA (from $0^{\circ}$ ) estimation in Fig. 4 when SNR is $15 \mathrm{~dB}$. The CI of the new method is narrower than that of [9] and [10].

At last, we implement simulations to verify the performance of the new method against difference of SNR of signals when $\sigma_{\beta}=40^{\circ}$, where the SNR of the signal from direction $-25^{\circ}$ is fixed to $20 \mathrm{~dB}$ and SNR of the other signal varies from $20 \mathrm{~dB}$ to $0 \mathrm{~dB}$. Simulation curves depicted in Fig. 3(b) verify that [9] tends to deteriorate with the difference of SNR increasing. The performance of [10] degrades substantially when the power difference is above $15 \mathrm{~dB}$ while the presented strategy still maintains considerable accuracy. Additionally, the two calibration methods have almost the same performance. The new calibration method performs better when the difference of SNR is less than $12 \mathrm{~dB}$ while the method in [5] only shows better performance when the difference of SNR is above $17 \mathrm{~dB}$.

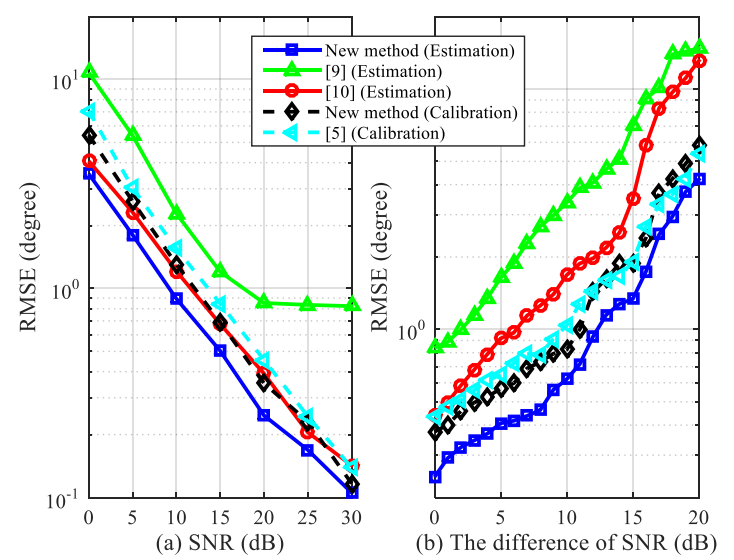

Fig. 3 RMSE against SNR and difference of SNR. (a) RMSE against SNR.

(b) RMSE against difference of SNR.
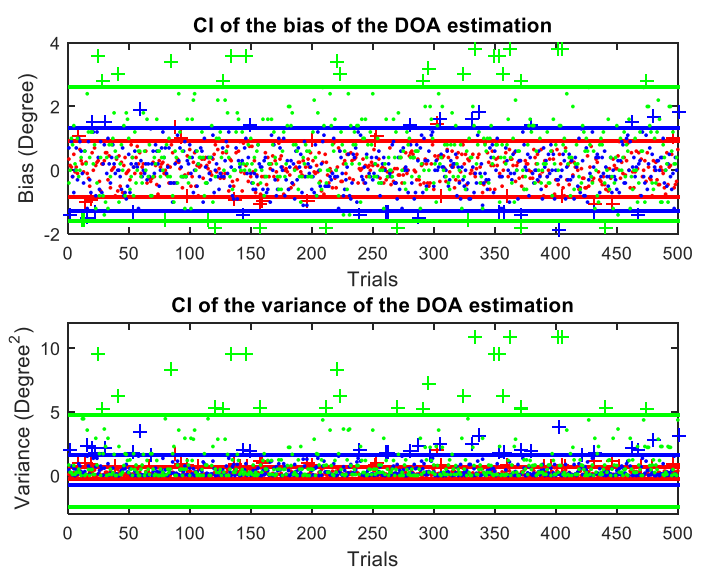

Fig. $4 \mathrm{CI}$ of the bias and variance of the DOA (from $0^{\circ}$ ) estimation of the new method (red), [9] (green) and [10] (blue) against the Monte Carlo trials. The lines are the lower and upper confidence limits. The point and plus represent the data in and out of the CI, respectively.

\section{CONCLUSION}

We propose a DOA estimation and sensor array error calibration method based on blind signal separation. The JADE algorithm is applied to separate the signal vector and mixing matrix. Based on a new mixing matrix and the reconstruction of the array output vector, we present a novel DOA 
estimation and sensor array error calibration method. The method presented is independent of array phase errors and performs well against difference of SNR of signals. Furthermore, this method requires fewer sensors than the methods in [9] and [10] when the number of signals is greater than two. Numerical simulations demonstrate that the new method is efficient and effective.

\section{References:}

[1]. Krim, H. and M. Viberg, Two decades of array signal processing research: the parametric approach. Signal Processing Magazine, IEEE, 1996. 13(4): p. 67-94.

[2]. Schmidt, R.O., Multiple emitter location and signal parameter estimation. Antennas and Propagation, IEEE Transactions on, 1986. 34(3): p. 276-280.

[3]. Roy, R. and T. Kailath, ESPRIT-estimation of signal parameters via rotational invariance techniques. Acoustics, Speech and Signal Processing, IEEE Transactions on, 1989. 37(7): p. 984-995.

[4]. Carlin, M., et al., Directions-of-Arrival Estimation Through Bayesian Compressive Sensing Strategies. IEEE Transactions on Antennas and Propagation, 2013. 61(7): p. 3828-3838.

[5]. Weiss, A.J. and B. Friedlander, Eigenstructure methods for direction finding with sensor gain and phase uncertainties. Circuits, Systems, and Signal Processing, 1990. 9(3): p. 271-300.

[6]. Paulraj, A. and T. Kailath. Direction of arrival estimation by eigenstructure methods with unknown sensor gain and phase. 1985: IEEE.

[7]. Li, Y. and M.H. Er, Theoretical analyses of gain and phase error calibration with optimal implementation for linear equispaced array. IEEE Transactions on Signal Processing, 2006. 54(2): p. 712-723.

[8]. Kim, J., et al., Blind Calibration for a Linear Array With Gain and Phase Error Using Independent Component Analysis. IEEE Antennas and Wireless Propagation Letters, 2010. 9: p. 1259-1262.

[9]. Liu, A., et al., An Eigenstructure Method for Estimating DOA and Sensor Gain-Phase Errors. IEEE Transactions on Signal Processing, 2011. 59(12): p. 5944-5956.

[10]. Cao, S., et al., A Hadamard Product Based Method for DOA Estimation and Gain-Phase Error Calibration. IEEE Transactions on Aerospace \&amp; Electronic Sy..., 2013. 49(2): p. 1224-1233.

[11].Cardoso, J.C.C.O. and A. Souloumiac, Blind beamforming for non-Gaussian signals. 1993, IET. p. 362--370.

[12]. Wylie, M.P., S. Roy and R.F. Schmitt. Self-calibration of linear equi-spaced (LES) arrays. 1993: IEEE.

[13]. Wu, H., et al., Theoretical Studies and Efficient Algorithm of Semi-Blind ICI Equalization for OFDM. IEEE TRANSACTIONS ON WIRELESS COMMUNICATIONS, 2008. 7(10): p. 3791-3798.

[14]. Levanon, N., Radar principles. New York, Wiley-Interscience, 1988, 320 p., 1988. 1. 